\title{
Investigation of tensile properties of RTV Silicone based Isotropic Magnetorheological Elastomers.
}

\author{
Bhaktha Sandesh ${ }^{1}$,Hegde Sriharsha ${ }^{2 *}$, Rao Sathish U. ${ }^{1}$ and Gandhi Nikhil ${ }^{2}$ \\ ${ }^{1}$ Department of Mechanical and Manufacturing Engineering, Manipal Institute of Technology, \\ Manipal Academy of Higher Education, Manipal- 576104, India \\ ${ }^{2}$ Department of Aeronautical and Automobile Engineering, Manipal Institute of Technology, Manipal \\ Academy of Higher Education, Manipal- 576104, India
}

\begin{abstract}
Magnetorheological elastomer (MRE) consists of an elastomer matrix and a Ferro-magnetic ingredient. The mechanical properties of MR elastomers can be reversibly controlled by applying a magnetic field of suitable intensity. The current work focusses on the enhancement of tensile property of RTV (Room Temperature Vulcanization) silicone based elastomer. The influence of Carbonyl iron powder (CIP) content and magnetic field were experimentally investigated. Addition of CIP increases the tensile modulus but it reduces the percentage elongation and tensile strength making it brittle. Under the influence of magnetic field, the enhancement of tensile properties up to $20 \%$ content was linear. The behavior above $20 \%$ is observed to be non-linear. The onset of non-linear stress-strain behavior is investigated. Regression equation is developed from the experimental data relating percentage content with the mechanical properties of MRE. The developed equation predicted the behavior of $27 \%$ MRE with an error of less than $8 \%$. Hyperelastic model developed by Yeoh was fitted to the stress-strain behavior of MRE with minimal error.
\end{abstract}

Keywords: Magnetorheological elastomer, RTV Silicone, Tensile testing, Regression Analysis, Hyperelastic modelling.

\section{Introduction}

Magnetorheological materials include MR fluids, MR elastomers and MR foams belong to a class of smart materials whose rheological properties can be controlled instantly and reversibly with the externally applied magnetic field. MR materials consists of Ferromagnetic particles suspended in a non- magnetic matrix such as liquid carrier fluid in case of MR fluid, elastomeric matrix in MR elastomer and a foam like matrix in MR foams [1, 2]. MR fluids and MR foams have a field dependent yield stress and MR Elastomers have a field dependent modulus $[1,2]$. The response time of MR fluids are fast but, fluids require a separate container for storage [2]. The sedimentation of the ferromagnetic ingredients over a period of time reduces the efficiency [3]. These limitations are overcome by employing magnetorheological elastomers.

\footnotetext{
*Corresponding author: sriharsha.hegde@manipal.edu
} 
Magnetorheological elastomers consists of micron sized Ferro-magnetic particles dispersed in a non-magnetic polymeric matrix. The particle are mixed before the curing process. When curing is done under a magnetic field, the interaction between the adjacent particles resulting in induced dipoles forming columnar structures parallel to the applied field. This imparts anisotropic nature to MRE $[1,4]$. These are also referred as structured MRE. Curing process without the application of magnetic field results in uniformly distributed particles within the matrix causing isotropic effect [5]. Both these type of MREs show significant enhancements in properties when tested under magnetic field $[1,4,6]$. Anisotropic MRE show better MR effect than isotropic samples [4, 5]. Lot of matrix materials are available for the processing of MRE [3]. RTV silicone rubber is the most widely used matrix by various researchers due to the ease of working in the laboratory and economical availability [2,4]. The working temperature of silicone elastomer is also wider than most matrix materials. Carbonyl iron powder is the obvious choice as ferromagnetic ingredient due to low coercivity and high saturation magnetization. The stiffness and the damping properties of the MRE is a function of the strength of the magnetic field $[4,7,8$, 9], size [10, 11], shape [12] and percentage content of carbonyl iron particles $[7,8,13,14]$. The aforementioned properties make MRE as semi - active /active suspension devices in various applications such as adaptive tuned vibration absorber (ATVA) [15], variable stiffness suspension bushing [16] etc. To successfully commercialize MRE, the mechanical characterization is necessary to ascertain its feasibility at different operating conditions.

Structured MRE display better stress-strain relationship than isotropic MRE. The maximum stress increases with increase in field strength [17]. Addition of Ferro-magnetic particles to the silicone rubber increases the tensile strength, stiffness and hardness. Anisotropic MREs aligned in the longitudinal direction have higher tensile strength and strain compared to transversely aligned MRE and isotropic MRE (27\% volume fraction) [18]. The addition of $20 \%$ CIP enhanced the tensile stress by $95 \%$. The elongation at break increased by $111 \%$ [19]. The tensile strength at break remains approximately constant up to $30 \%$ volume of CIP. Further increase in CIP content deteriorates the mechanical properties [20].

In the present work, RTV-Si based MRE samples were prepared by varying the percentage content of CIP. The stress-strain behavior of the samples were tested under varying magnetic fields. Hyper-elastic model (Yeoh model) was fitted to the experimental stress-strain data. Regression equations relating the Young's modulus, tensile strength and the percentage elongation as a function of \%CIP content was developed. The relation was used to predict the properties of $27 \%$ CIP content MRE which was experimentally validated. The error was found to be within acceptable limits.

\section{Methodology}

\subsection{Sample preparation}

A two part Silicone RTV rubber from Dow Corning Corporation, India (Silastic 3483 base and Silastic 83 curing agent) was used as matrix. CIP of average diameter $6.23 \mu \mathrm{m}$ (Chengdu Nuclear 857 New materials Ltd, China) was employed as Ferro-magnetic ingredient. In the first step, the CIP was mixed with the elastomer base and stirred thoroughly to ensure uniform distribution. The curing agent was mixed then it was stirred again and the mixture was kept inside a vacuum chamber to remove air bubbles. The mixture is poured in to acrylic mould (dimensions as per ASTM D412 - Die C) and 
allowed to cure for a period of 24 hours. Samples with $0 \%, 5 \%, 10 \%, 15 \%, 20 \%, 25 \%$ and $30 \%$ CIP were prepared. One of the test samples is shown in figure below (Fig. 1).

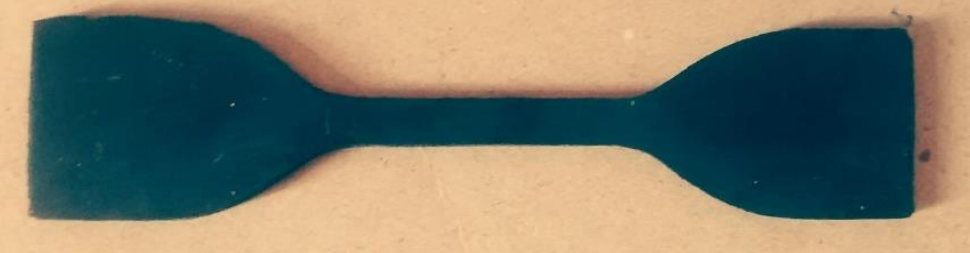

Fig. 1. Tensile test sample

\section{2 Magnetic field analysis}

Rare earth Neodymium permanent magnets were used in the form of Halbach array to produce magnetic field (Fig. 2). An FEMM (Finite Element Method Magnetics) analysis of the same was conducted to ensure uniform distribution of magnetic field (Fig. 3a). The variation of the field with respect to the gap between the poles shown in figure $3 \mathrm{~b}$ indicates that approximately $0.5 \mathrm{~T}$ field is produced at the center. This was validated using a Gaussmeter (SES instruments, Roorkee).

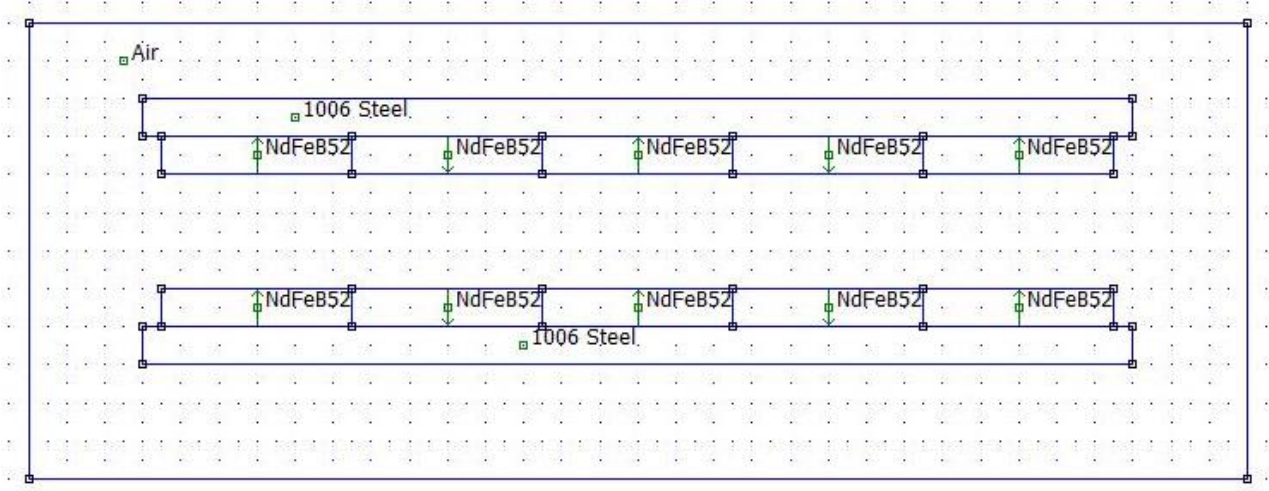

Fig. 2. Arrangement of permanent magnets 


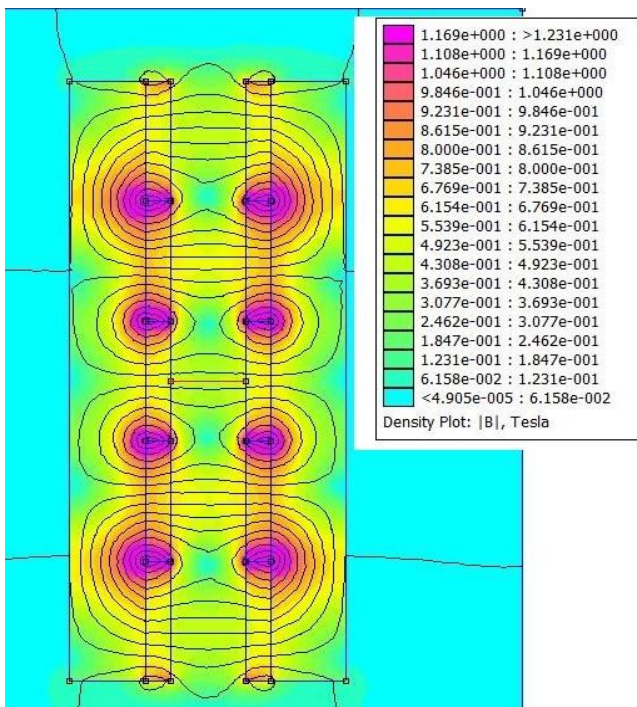

Fig.3a. The flux density of Halbach array

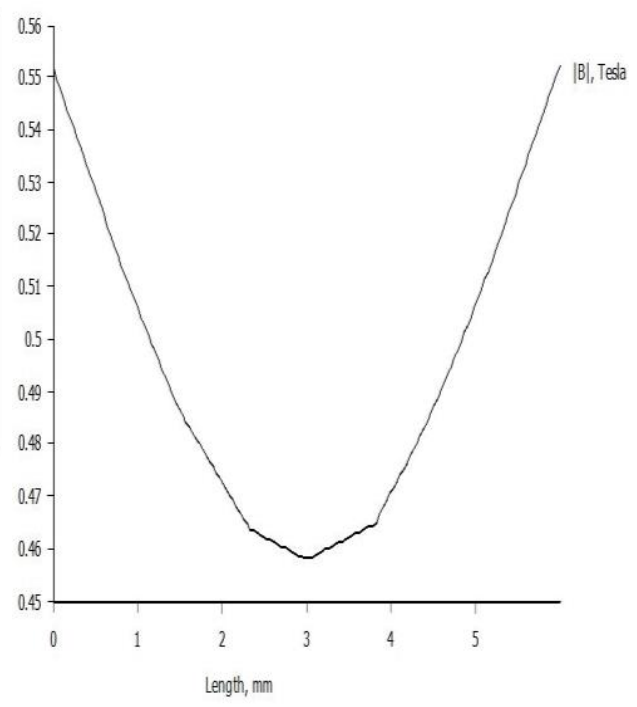

Fig.3b. Flux density v/s distance

\subsection{Tensile test}

Tensile test was performed on a Universal testing machine (INSTRON 3366) both in the presence and absence of a magnetic field. The magnetic field was applied using Halbach array along the gauge length $(33 \mathrm{~mm})$ of the test specimen. The samples were tested at a cross head speed of $500 \mathrm{~mm} / \mathrm{min}$ as per standard ASTM D412 [21] at room temperature. The photograph of the test set up is depicted in Fig. $4 \mathrm{a}$ and $4 \mathrm{~b}$.

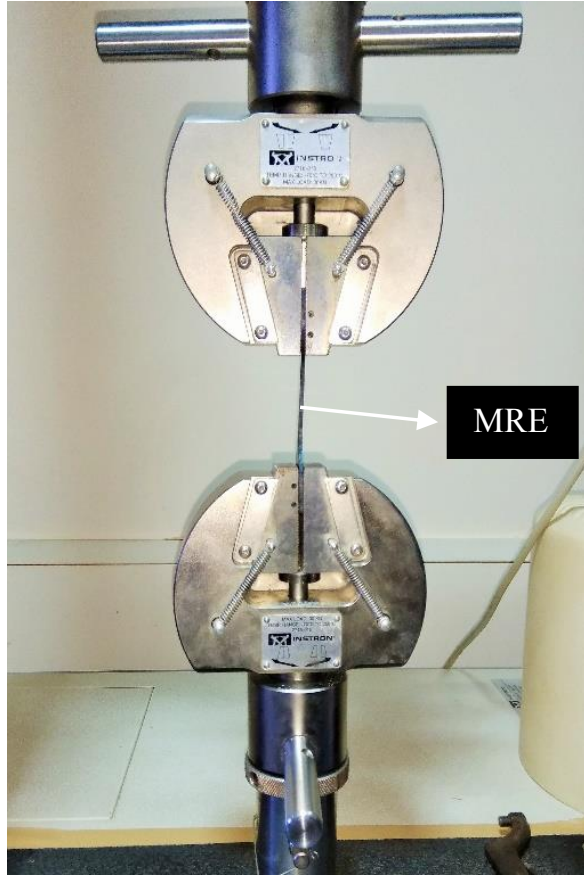

Fig.4a. Tensile testing without magnets

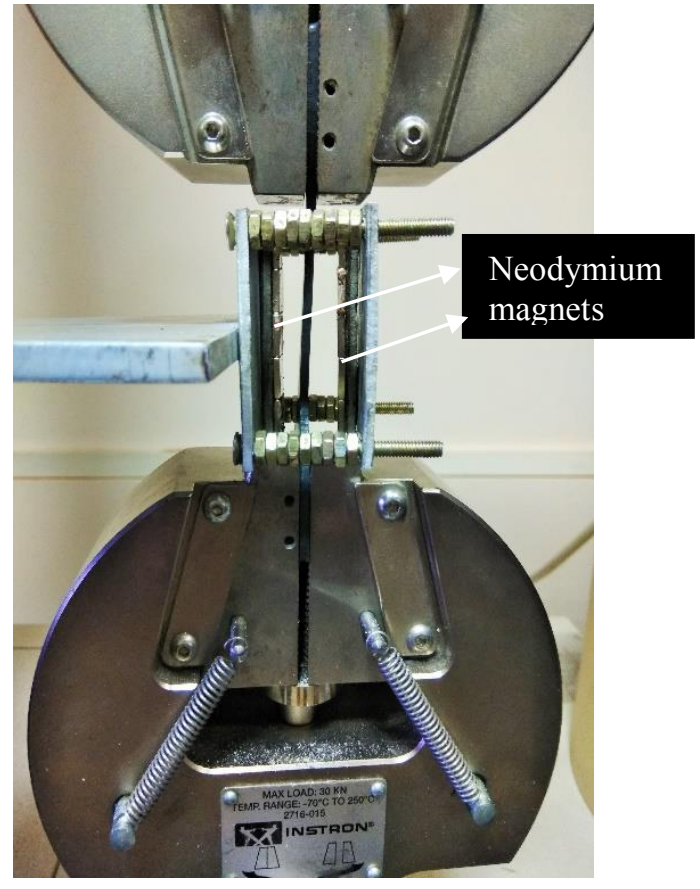

Fig.4b. Tensile test under magnetic field 


\subsection{Hardness measurement}

The variation of hardness of the test samples with and without the application of the magnetic field was measured by a Shore (A) durometer as per ASTM D2240 [22]. The hardness is measured at 5 different locations and the averaged valued is considered for analysis.

\subsection{Hyperelastic modelling}

Hyperelastic material models are used to explain the nonlinear elastic behavior of rubber like materials under the assumptions of material isotropy and incompressibility. These constitutive models mainly rely on the definition of strain energy functions which is governed by the values of strain invariants. Silicone rubber used in the current study exhibit hyperelastic characteristics $[23,24]$ and this behavior is modelled using the constitutive equations. The Yeoh model [25] is used in this study since the quality of the fit was observed to be good. The quality of fit was ensured by coefficient of determination $\left(\mathrm{R}_{2}\right)$ whose value is required to be close to 1 which meant that the difference between the experimental value and model's predicted value are small. The equation showing the relationship between the strain energy and the strain invariants for this model could be represented as

$$
\psi=\sum_{i=1}^{N} C_{i 0}\left(I_{1}-3\right)^{i}
$$

where

$\psi=$ Strain energy density

$I_{l}=$ First variant of C

$C=$ Right Cauchy-Green def tensor

$C_{i 0}=$ Stress like parameters defining stiffness of material

$N=$ Number of terms

Stress-strain data of all the samples obtained from experiments was imported to Hyperfit freeware and curve fitting was done for Yeoh model $(N=3)$. Stress-strain curves were generated based on the values of the strain variants obtained with averaged $R_{2}$ value being 0.998 .

\section{Results and discussions}

\subsection{Stress-strain behavior}

The Fig. 5 shows the variation of stress-strain (average data of 3 samples each obtained from force-displacement graph) behavior of the samples tested in the absence of the magnetic field. From the stress-strain graph it is observed that the pure silicone rubber sample exhibited good ductility where the stress increased with the increase in the strain. As the percentage CIP content was increased, the reinforcing effect increases the stiffness of the matrix. When the ingredients are absent, the cross-linking of the polymer chain is more continuous rendering it more elastic. With the increase in percentage content the continuity of the polymer chain breaks making the elastomer more brittle resulting in a decrease in elongation at break. The addition of Carbonyl iron particles to the matrix 
decreased the tensile strength (2.25 MPa for pure silicone and 1.37 MPa for 30\% MRE) due to the increase in the brittleness of the MRE. However, the tensile modulus increases with the increase in the percentage CIP in a nonlinear manner which indicates that the stiffness of the MRE increases. The increase in the stiffness is theoretically quantified by hyperelastic modelling. The variation of tensile properties with respect to the change in the percentage carbonyl iron powder content have been shown in the Table 1.

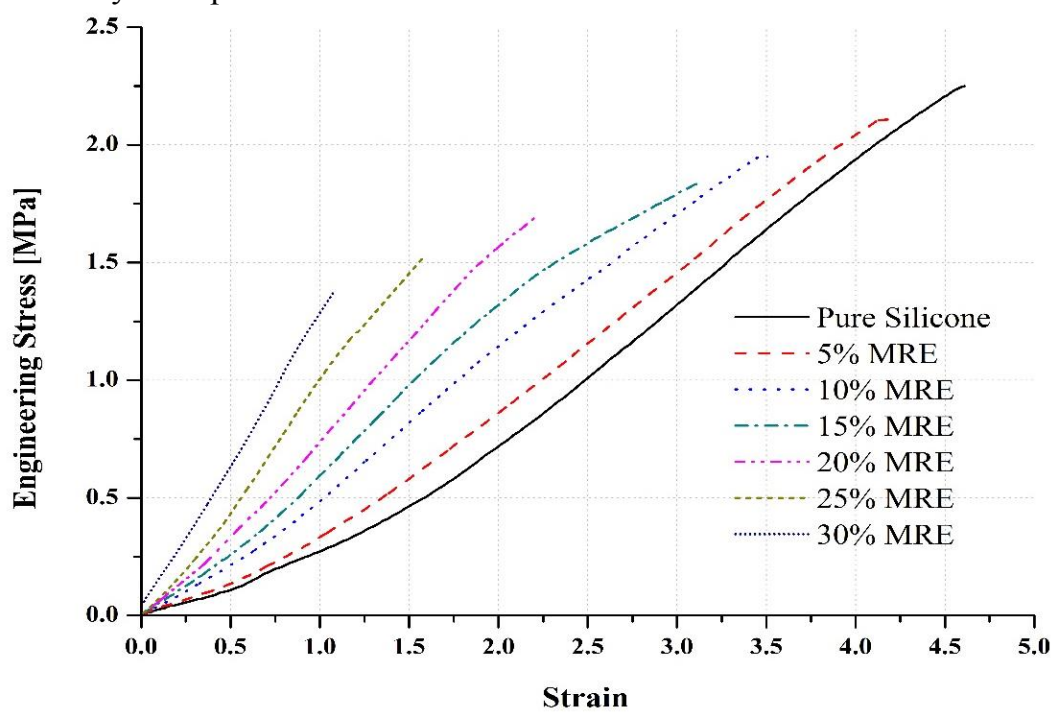

Fig. 5. Stress v/s Strain behavior of isotropic MREs at 0T.

Table1. Tensile Properties of MRE

\begin{tabular}{cccc}
\hline $\begin{array}{c}\text { Percentage } \\
\text { volume of CIP }\end{array}$ & $\begin{array}{c}\text { Tensile strength, s } \\
(\mathbf{M P a})\end{array}$ & $\begin{array}{c}\text { Tensile modulus, } \\
\text { E (MPa) }\end{array}$ & \% Elongation \\
\hline 0 & 2.25 & 0.62 & 461.1 \\
5 & 2.11 & 0.75 & 417.9 \\
10 & 1.95 & 0.84 & 350.72 \\
15 & 1.83 & 0.94 & 314.2 \\
20 & 1.69 & 1.03 & 223.1 \\
25 & 1.59 & 1.17 & 152.7 \\
30 & 1.37 & 1.39 & 107.6 \\
\hline
\end{tabular}




\subsection{Stress-strain behavior under the influence of magnetic field}
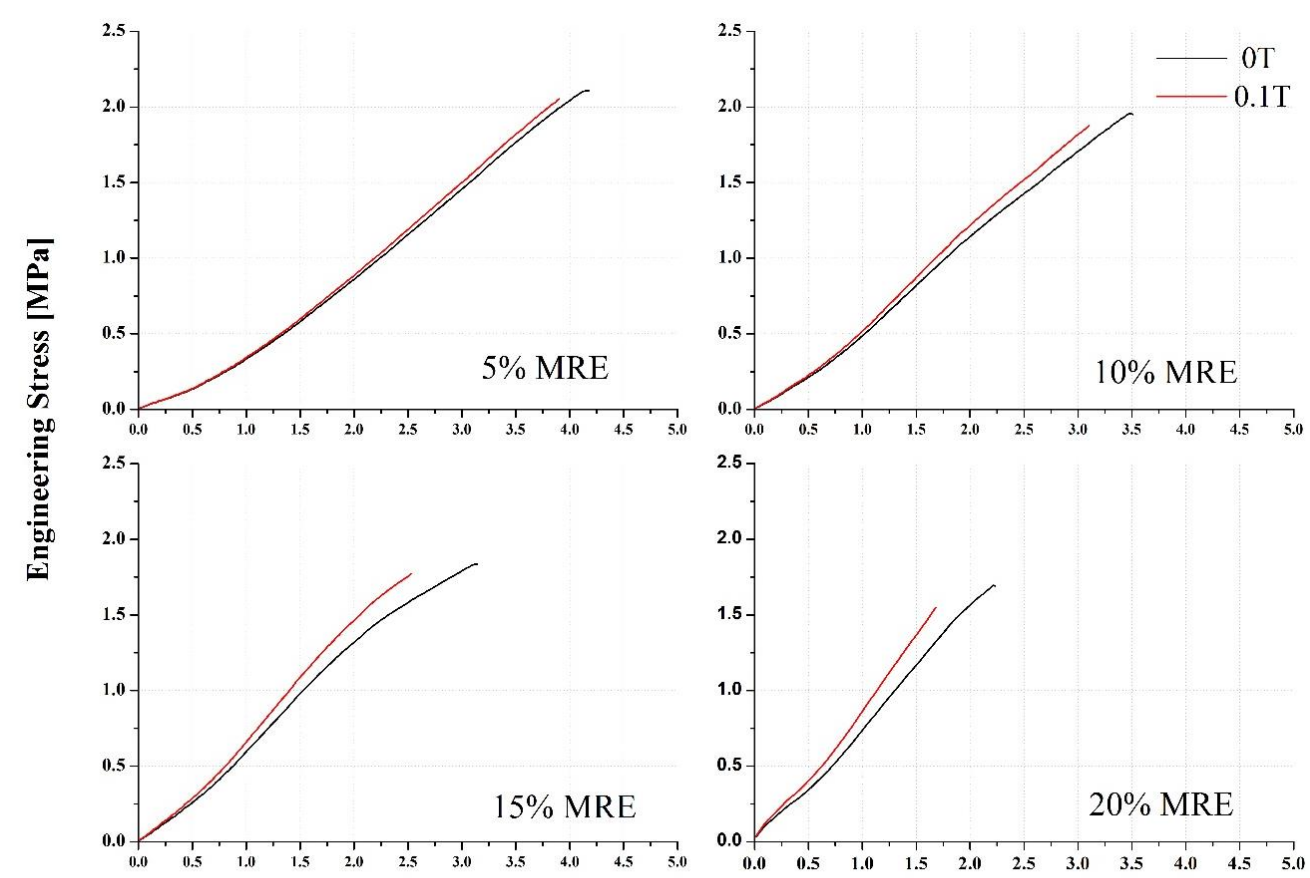

\section{Strain}

Fig. 6. Stress-strain behavior of MRE with magnetic field

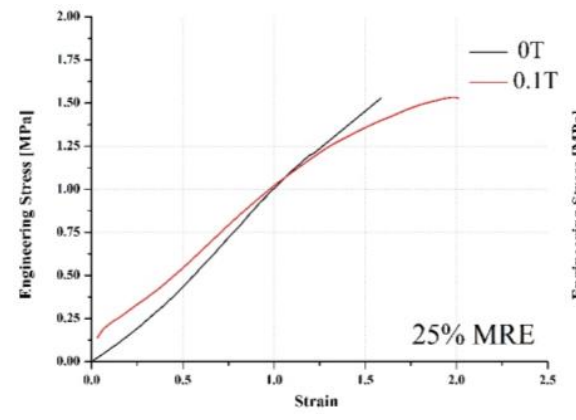

Fig.7a. Stress strain behavior $25 \% \mathrm{MRE}$

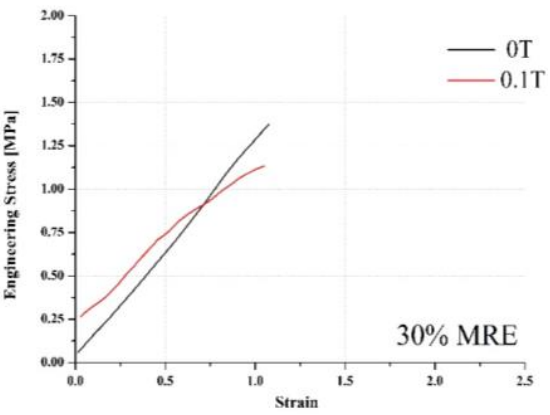

Fig.7b. Stress strain behavior 30\% MRE

The elongation of pure sample at break is about $460 \%$ which is more than MRE samples. The addition of CIP is resulting in improved Young's modulus, but the elongation at break is reducing. The trend is uniform across all samples under the absence of magnetic field. When the field is applied, the behavior is similar, but with an improved field induced modulus resulting from hardness increase up to $20 \%$ CIP concentration. The ductility is higher at lower CIP content and the distance between the adjacent particles is also relatively higher. As the strain is increased, the distance between the adjacent particle increases. This behavior is linear if the matrix is relatively more ductile. For $25 \%$ and $30 \%$ CIP samples, the field induced behavior is slightly different resulting from non- linearity. The $25 \%$ sample starts to show non-linear behavior at about $100 \%$ strain at $0.1 \mathrm{~T}$ (figure $7 \mathrm{a}$ ). This is lower than the samples with lower CIP content. Even though, the elongation is slightly 
improved when compared to $0 \mathrm{~T}$ the linearity is compromised. The onset of non-linearity for $30 \%$ CIP sample is further reduced at approximately at $75 \%$ strain (Fig. $7 \mathrm{~b}$ ).

The phenomenon of on-set of non- linearity can be explained by considering a unit cell of MRE with 4 CIP particles for lower percentage content of CIP (0-20\% CIP samples) (Fig. 8) and 6 CIP particles for higher percentage (Fig. 9) content of CIP (25\% and 30\% CIP samples). When the MRE is subjected tensile force in the longitudinal direction, equal and opposite force acts at the fixed end which resists the tensile loading increasing the modulus. During tensile loading the CIP particles in the longitudinal direction tend to move away from each other. However, particles in the lateral direction come closer which prevents further elongation in the longitudinal direction. With the application of magnetic field, the distance between the particles decreases in the lateral direction due to the dipole. The dipole attraction between the adjacent particles in the lateral direction results in increased resistance to tensile force increasing the tensile modulus. For lower percentage of CIP samples, the increase is not enough to cause non-linearity. For higher percentage of CIP samples the gap between the particles is lower which further prevents the elongation in the longitudinal direction making it more brittle and reducing the tensile strength. Further, under the influence of magnetic field, the force of attraction between the particles in the lateral direction is higher causing increased resistance to the loading which accounts for increase in tensile modulus for lower values of strain. However, at higher values of strain, since the distance between the adjacent particles is considerably small, the resistance to the tensile force exerted by the sample decreases. The sample is highly brittle such that the effect of the magnetic field is arrested reducing the tensile modulus. This results in nonlinear behavior.

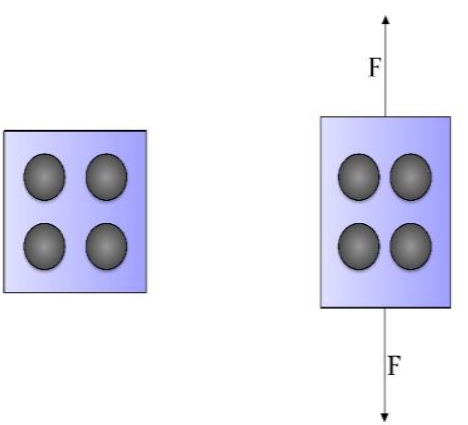

Fig. 8a. Behaviour under tensile force

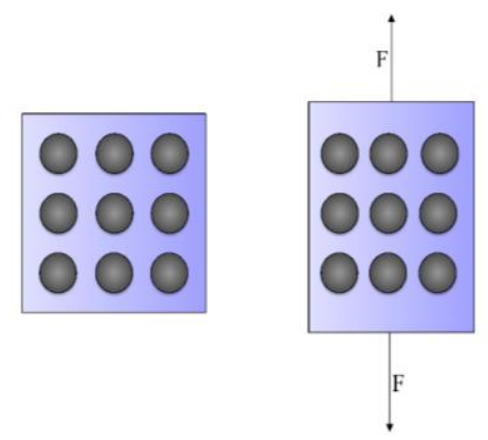

Fig. 9a. Behaviour under tensile force

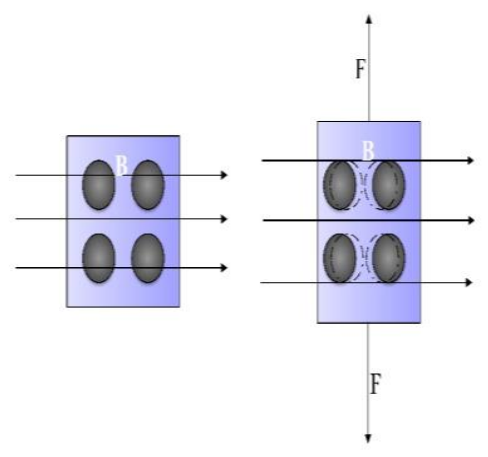

Fig. 8b. Behaviour under magnetic field

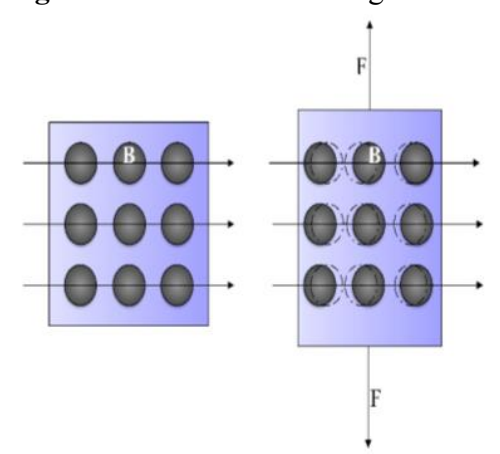

Fig. 9b. Behaviour under magnetic field 


\subsection{Hyperelastic modelling}

The material constants determined for pure silicone and MRE samples are listed in Table 2. It is observed from the table that with the increase in percentage content of CIP, the value of $C_{10}, C_{20}$ and $C_{30}$ increases. The greater the value of $C_{\mathrm{i} 0}$, higher is the stiffness of the sample. Silicone reinforced with $30 \%$ CIP has the highest value of $C i_{0}$, which indicates that this sample is the hardest among all the samples. Pure silicone rubber has the lowest value of $C_{\mathrm{i} 0}$, which indicates that the percent elongation is highest among all samples. It can be concluded that the addition of the CIP has an effect on the stiffness of the samples which in turn effects the tensile properties.

Table 2. Parameters of Yeoh model

\begin{tabular}{cccc}
\hline \multicolumn{4}{c}{ Yeoh Model $(\mathbf{N}=\mathbf{3})$} \\
\hline \% CIP & $\mathbf{C}_{\mathbf{1 0}}$ & $\mathbf{C}_{\mathbf{2 0}}$ & $\mathbf{C}_{\mathbf{3 0}}$ \\
\hline 0 & 0.06 & 0.005 & 0 \\
5 & 0.078 & 0.006 & 0 \\
10 & 0.115 & 0.008 & 0 \\
15 & 0.125 & 0.012 & 0 \\
20 & 0.148 & 0.018 & -0.001 \\
25 & 0.163 & 0.041 & -0.004 \\
30 & 0.256 & 0.044 & -0.005 \\
\hline
\end{tabular}

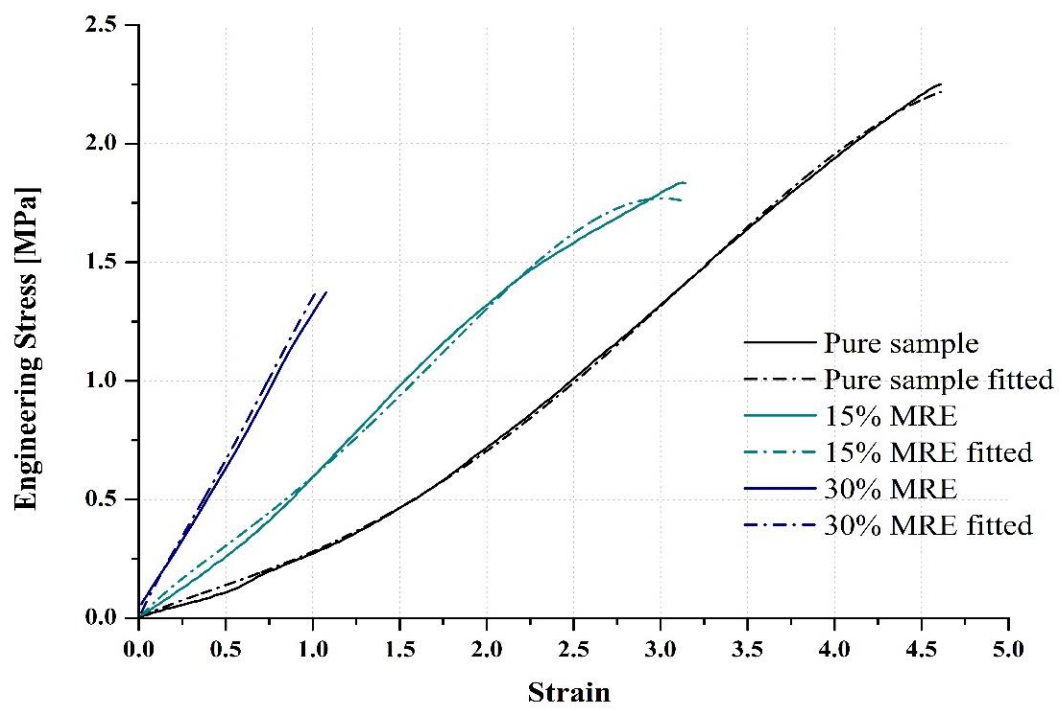

Fig.10. Yeoh model ( $\mathrm{N}=3$ ) fitted for samples having 0, 15 and 30\% CIP

Fig. 10 shows the Stress-strain characteristics of MREs having 0, 15 and 30\% CIP and their corresponding Yeoh Model $(\mathrm{N}=3)$ fitted plots. Selected graphs are shown here for the clarity and other samples also show a similar trend. 


\subsection{Hardness}

From the Table 3, it is observed that, the addition of CIP enhances the hardness due to reinforcing effect. The hardness of the MRE samples increase with the magnetic field due to the alignment of the CIP along the flux lines. This trend is consistent for all the MRE samples.

Table 3. Effect of addition of CIP and application of magnetic field on the hardness of the MREs.

\begin{tabular}{ccc}
\hline & \multicolumn{2}{c}{ Hardness (Shore A) } \\
\cline { 2 - 3 } Percentage volume of CIP & Without magnetic field & With magnetic field (0.1T) \\
\cline { 2 - 3 } 5 & 9 & 9 \\
10 & 10 & 12 \\
15 & 12 & 14 \\
20 & 16 & 20 \\
25 & 22 & 26 \\
30 & 29 & 32 \\
& 33 & 36
\end{tabular}

\subsection{Regression equation}

Regression analysis is a statistical tool used to determine the relationship between two variables. It is used to determine the effect of the input parameters on the output. In this study, the effect of percentage volume of CIP on the tensile strength, tensile modulus and \% elongation is analytically determined using regression equations. The regression equations are generated based on the experimental results. A second order polynomial is used in this study since the correlation coefficient $\left(\mathrm{r}^{2}\right)$ was observed to be close to 1 . Table 4 shows the Regression equation to predict Tensile strength, Tensile modulus and percentage elongation. Using these equations, the tensile parameters are calculated for $27 \%$ volume of CIP. The stress-strain behavior of 25\%, 27\% and 30\% MRE samples is depicted in Fig. 11.

Table 4. Regression equation and Correlation coefficient for tensile parameters

\begin{tabular}{clc}
\hline Response & \multicolumn{1}{c}{ Regression Equation } & $\begin{array}{c}\text { Correlation } \\
\text { coefficient } \\
\left(\boldsymbol{r}^{\mathbf{2}}\right)\end{array}$ \\
\hline Tensile Strength & $R_{T S}=\left(2.24095-(0.02614 * X)-\left(6.67 * 10^{\left.-5 * X^{2}\right)}\right.\right.$ & 0.99 \\
Tensile Modulus & $R_{T M}=\left(0.64609+(0.01392 * X)+\left(3.3981 * 10-4 * X^{2}\right)\right.$ & 0.98 \\
$\%$ Elongation & $R_{E}=\left(465.72-(10.35 * X)-\left(0.0641 * X^{2}\right)\right.$ & 0.98 \\
\hline
\end{tabular}

where $X=$ Percentage volume of CIP 
Table 5 shows the percentage error between the experimental values and predicted value generated using the regression equations. The average error for the model is observed to be less than $6 \%$ which showed a good correlation between the experimental values and predicted values.

Table 5. Calculated and Experimental value comparison for 27\% MRE

\begin{tabular}{|c|c|c|c|c|c|c|}
\hline \multirow{2}{*}{ Parameters } & \multirow{2}{*}{$\begin{array}{l}\text { Predicted } \\
\text { values }\end{array}$} & \multicolumn{4}{|c|}{ Experimental Values } & \multirow[t]{2}{*}{$\%$ Error } \\
\hline & & Trial 1 & Trial 2 & Trial 3 & Average & \\
\hline $\begin{array}{c}\text { Tensile Strength } \\
(\mathrm{MPa})\end{array}$ & 1.48 & 1.54 & 1.57 & 1.56 & 1.55 & 4.7 \\
\hline $\begin{array}{l}\text { Tensile Modulus } \\
\quad(\mathrm{MPa})\end{array}$ & 1.27 & 1.30 & 1.32 & 1.33 & 1.31 & 3.14 \\
\hline$\%$ Elongation & 139.54 & 147.37 & 152.99 & 149.91 & 149.9 & 7.42 \\
\hline
\end{tabular}

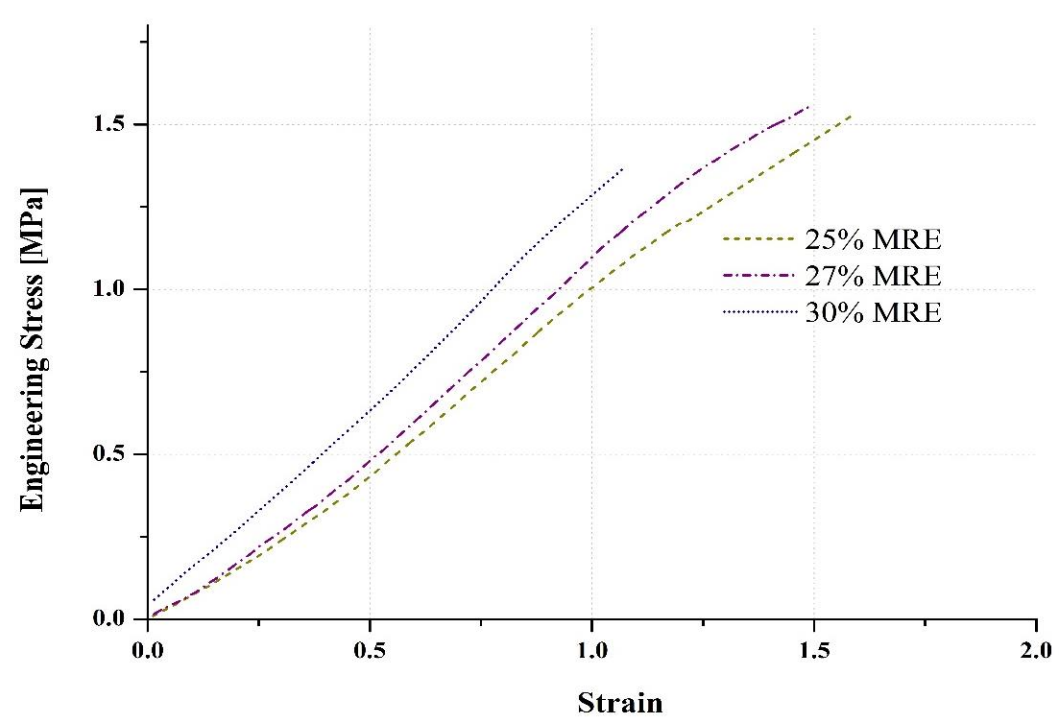

Fig.11. Stress v/s Strain behavior of $27 \%$ MRE

\section{Conclusions}

RTV silicone based MRE samples were prepared with different CIP percentage content. Tensile and hardness tests were conducted at varying magnetic fields, which resulted in the following conclusions.

1. The addition of CIP improves modulus of the MRE compared to pure silicone sample. The ductility of the samples reduces with the percentage increase in the CIP content. The elongation at break for pure sample is $460 \%$ which reduces to $107 \%$ for $30 \%$ MRE. Under the influence of magnetic field, the stress strain behavior is improved and up to $20 \%$ CIP, the behavior is linear. Higher percentage CIP samples showed nonlinear behavior under the influence of the field. 
2. The addition of CIP increases the hardness due to reinforcement effect. The application of magnetic field further increases the hardness.

3. Stress-Strain behavior of MRE was employed to Yeoh hyper-elastic modelling. The model was fitted to experimental data with negligible errors.

4. The regression equation was developed which can predict the modulus, tensile strength and percentage elongation as a function of CIP percentage content. The equation predicted the behavior of $27 \%$ content CIP with good effect.

\section{References}

1. J. D. Carlson and M. R. Jolly, Mechatronics, 10, 555-569 (2008).

2. Weihua Li and X. Zhang, Recent Patents on Mechanical Engineering, 1,161-166 (2008).

3. Y. Li, J. Li, W. Li and H. Du, Smart Mater. Struct, 23, 1-24, (2014).

4. J. M. Ginder, M. E. Nichols, L. D Elie, and J. L. Tardiff, Smart Mater. Struct., 131-138 (1991).

5. X. L. Gong, X. Z. Zhang, and P. Q. Zhang, Polym Test., 24, 669-676 (2005).

6. M. Lokander and B. Stenberg:, Polym Test., 22, 677-680 (2003).

7. L. Chen, X. L. Gong, and W. H. Li, Chin. J. Chem. Phys, 15, 271-274(2008).

8. M. R. Jolly, J. D. Carlson, and B. C. Munoz, J. Intel. Mat. Syst. Str., 7, 612-622 (1996).

9. J. F. Li and X. L. Gong, J. Cent. South Univ., 15, 261-265 (2008).

10. S. Hegde, U. R. Poojary, and K. V. Gangadharan, International Conference on Advances in Manufacturing and material engineering. Mangalore, India, 2301-2309 (2014).

11. Y.C. Fan, X. Gong, S. Xuan, W. Zhang, J. Zheng, and W. Jiang, Smart Mater. Struct., 20, 1-8 (2011).

12. H. J. Song, O. Padalka, M. Werely and R. C. Bell, 50th AIAA/ASME/ ASCE/ AHS/ ASC Strucutres, Structural Dynamics, and materials conference, Palm springs, USA, AIAA, 2009-2118 (2009).

13. A. Boczkowska and S. F. Awietjan, J. Mater. Sci., 44, 4104-4111 (2009).

14. L. C. Davis, J. App. Phys., 85, 3348-3351(1999).

15. H. X. Deng and and X. L. Gong, J. Intel. Mat. Syst. Str., 18, 1205-1210 (2007).

16. J. R. Watson, Patent US5609353, 1997.

17. C. Bellan and G. Bossis, Int. J. Modern Phys., 16, 2447-2453 (2002).

18. M. Farshad and Andre Benine, Polym. Test., 23,347-353 (2004).

19. I.A.P Martinez, L.M.P Pineda, L.M.L Sanchez, O.S Romero, J.G.P. Cordova and A.E Zuniga, Polym. Test., 57, 78-86 (2017).

20. Lokander M and B Stenberg, Polym Test., 22, 245-251(2003).

21. ASTM D $412-15 \mathrm{a}, 2013$, Standard test methods for Vulcanized rubber and Thermoplastic Elastomers - Tension, American society for Testing and Materials, United States

22. ASTM D 2240 - 15, 2017, Standard Test method for rubber property - Durometer Hardness, American society for Testing and Materials, United States 
23. V. Vahapoglu, S. karadeniz, and I Yazici, Experimental techniques, 35, 17-23(2011).

24. M. Noor, S.N. Azizzat, and J. Mahmud, Advanced materials research, 915, 858-866 (2014).

25. OH. Yeoh, Rubber chemistry and Technology, 66, 754-771(1993). 